ISSN 1997-5902

\title{
Influence de l'association culturale sur la capacité de nodulation de trois espèces de légumineuses : Arachide, Niébé et Soja vert
}

\author{
KOUAME N'guessan ${ }^{1 *}$, KOUASSI N'dri Jacob ${ }^{1}$, AYOLIE Koutoua ${ }^{1}$, YAO Kouassi Blaise ${ }^{1}$ et \\ YATTY Kouadio Justin 1 \\ Université Jean Lorougnon GUEDE, Laboratoire d'amélioration de la production agricole, UFR Agroforesterie, BP \\ 150 Daloa Côte d'Ivoire. \\ *Auteur correspondant : (225) 09240126 ; (225) 062545 10, e-mail : maximekouamelma@yahoo.fr, BP 150 \\ Daloa, (225) Côte d'Ivoire. \\ Original submitted in on $5^{\text {th }}$ November 2019. Published online at www.m.elewa.org/journals/ on $31^{\text {st }}$ January 2020 \\ https://doi.org/10.35759/JABs.v145.8
}

\section{RESUME}

Objectifs : Les légumineuses ont des rôles de protection des sols, de lutte contre les adventices et de l'amélioration de la fertilité par la fixation de l'azote atmosphérique. Cette fixation de l'azote atmosphérique se fait grâce aux souches de Rhizobium contenues dans leurs nodosités. Cette étude vise donc à promouvoir une pratique culturale pouvant améliorer la capacité de nodulation de ces légumineuses.

Méthodologie et résultats : L'étude a porté sur la culture des trois légumineuses en culture pure et en association culturale et s'est déroulée à l'université Jean Lorougnon GUEDE de Daloa, Côte d'Ivoire. Cette étude a adopté un dispositif expérimental en blocs complètement randomisés. Les paramètres mesurés ont concerné le nombre de nodules, la longueur et le poids de la plante, la longueur et le poids du système racinaire. Les résultats ont montré que l'association des légumineuses ne favorise pas la capacité de nodulation de celles-ci.

Conclusion et application de la recherche : Les légumineuses sont très appréciées pour leurs vertus alimentaires, agronomiques et économiques. Par contre, les sols ivoiriens sont caractérisés par une tendance à l'acidification et à une baisse de la teneur en éléments fertilisants tels que le phosphore et l'azote qui ont une incidence directe sur les rendements. Pour restaurer la fertilité du sol, il faut donc intensifier la nodulation de ces légumineuses en choisissant une option de pratiques culturales adéquates.

Mots clés : légumineuses, azote, phosphore, nodulation, pratique culturale.

\section{ABSTRACT}

Influence of cultural association on the nodulation capacity of three legume species: groundnuts, cowpeas and green soybeans

Objectives: Legumes have roles in protecting the soil, controlling weeds and improving fertility by fixing atmospheric nitrogen. This fixation of atmospheric nitrogen is done thanks to the Rhizobium strains found in their nodules. This study therefore aims to promote a cultivation practice that can improve the nodulation capacity of these legumes.

Methodology and results: The study focused on the cultivation of the three legumes in pure culture and in cultural combination and took place at the university Jean Lorougnon GUEDE of Daloa, Côte d'Ivoire. This study adopted an experimental device in completely randomized blocks. The parameters measured included the number of nodules, the length and weight of the plant, the length and weight of the root 
system. The results showed that the association of legumes does not favor the nodulation capacity of these legumes.

Conclusion and application of research: Legumes are highly valued for their nutritional, agronomic and economic value. On the other hand, Ivorian soils are characterized by a tendency towards acidification and a decrease in the content of nutrients such as phosphorus and nitrogen, which have a direct impact on yields. To restore soil fertility, we must intensify the nodulation of these legumes by choosing an appropriate cultural practice option.

Keywords: legumes, nitrogen, phosphorus, nodulation, cultural practice.

\section{INTRODUCTION}

Les légumineuses forment une des plus grandes familles des plantes à fleurs appartenant à la famille des Fabacées. Elles jouent un triple rôle de protection des sols contre la dégradation, la lutte contre les adventices, l'amélioration et le maintien de la fertilité des sols par la fixation de l'azote atmosphérique (Akakpo et al., 1999). En produisant des grains et des fourrages de qualité, la culture des légumineuses peut augmenter le revenu brut des exploitations en réduisant les charges en engrais notamment grâce à la fixation symbiotique de l'azote atmosphérique (Carsky et al., 2003). Parmi ces légumineuses, le niébé (Vigna unguiculata $(\mathrm{L})$ walp), l'arachide (Arachis hypogaea) et le soja vert (Vigna radiata) sont les plus importants dans la plupart des communautés africaines où ils sont cultivés (Taffouo et al., 2008). Elles exercent une influence favorable sur la fertilité des sols grâce à

\section{MATERIEL ET METHODES}

Site d'étude : L'étude a été réalisée sur une parcelle expérimentale dans la localité de Daloa. La ville de Daloa est localisée dans la région du haut Sassandra au Centre Ouest de la Côte d'Ivoire entre le $6^{\circ}$ et le $7^{\circ}$ de latitude Nord et le $7^{\circ}$ et $8^{\circ}$ de longitude Ouest. Cette région a une superficie de $15200 \mathrm{~km}^{2}$ pour une population estimée à 1.430 .960 habitants ( $\mathrm{Ba}, 2014)$. Le département de Daloa est délimité au Nord par les départements de Vavoua et de Zuénoula, au Sud par ceux d'Issia et de Sinfra, à l'ouest les circonscriptions départementales de Duékoué et de Bangolo et à l'Est par celle de Bouaflé (Figure 1). Le climat a quatre saisons. La grande saison des pluies part d'avril à mijuillet, la petite saison sèche de mi-juillet à miseptembre, la petite saison des pluies de miseptembre à mi-novembre et la grande saison sèche la symbiose fixatrice d'azote avec les souches de rhizobium. Elles jouent par conséquent un rôle primordial dans la rotation des cultures (Baudoin et al., 2001). Aussi, les sols ivoiriens sont caractérisés par une baisse du taux de la matière organique, une désaturation du complexe absorbant et une acidification. De plus, une forte pression est exercée par les agriculteurs sur le patrimoine foncier pour pouvoir répondre aux besoins d'une population de plus en plus grandissante. Ce qui engendre un déclin du taux d'azote dans les sols. Une alternative pour restaurer la fertilité est la fixation biologique de l'azote par les rhizobiums en symbiose avec les légumineuses. L'objectif global de cette étude est de promouvoir la pratique culturale pouvant améliorer la capacité de nodulation des légumineuses.

de décembre à mars. Les saisons sèches et humides alternent avec les températures variant de $24,65^{\circ} \mathrm{C}$ à $27,75^{\circ} \mathrm{C}$ en moyenne. La pluviométrie, la température et l'humidité moyenne caractérisant le site d'étude dans les périodes d'essai de Mai à Août 2016 correspondant aux grandes saisons des pluies sont respectivement: $142,81 \mathrm{~mm} ; 26,42^{\circ} \mathrm{C}$ et 83,7 . Le substrat pédologique de Daloa appartient au vieux socle précambrien composé de granite. Les sols de la région sont majoritairement ferralitiques (typiques). Ils sont généralement très profonds avec un taux élevé de matière organique. II s'agit de sols ferralitiques d'origine granitique moyennement à faiblement désaturés. Ces sols ferralitiques présentent de bonnes aptitudes agricoles et se prêtent à tous les types de culture (Atlas, 2007). 


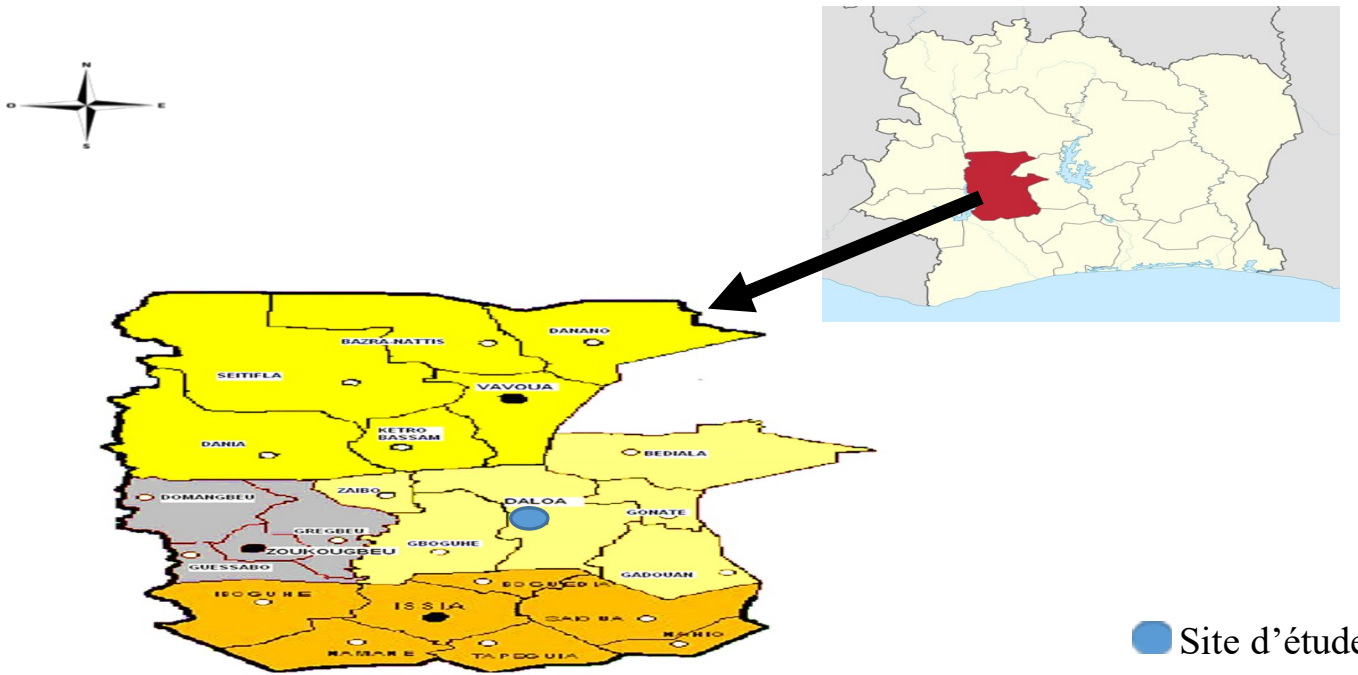

Figure 1: Présentation de la zone d'étude

Matériel végétal : Le matériel végétal utilisé dans cette étude a été constitué des graines de trois espèces de légumineuses issues de la collection de
l'Université jean Lorougnon Guédé. II s'agit entre autre de Vigna unguiculata, Vigna radiata et d'Arachis hypogaea (Figure 2).

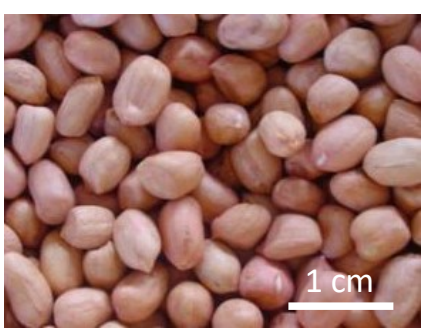

a

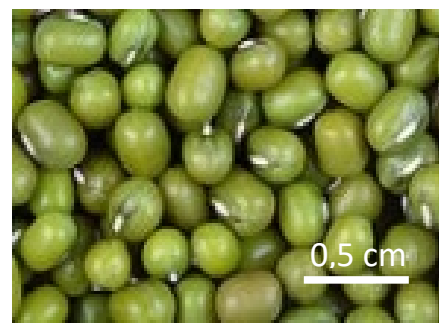

b

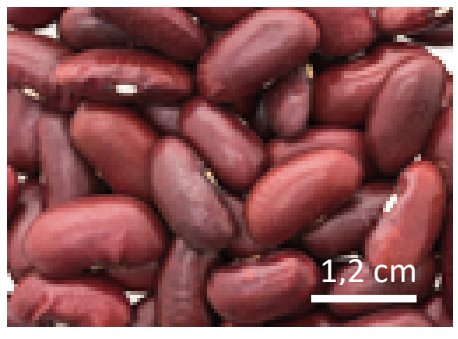

c

Figure 2 : Matériel végétal

(a) Arachide (Arachis hypogaea), (b) Soja vert (Vigna radiata) et (c) niébé (Vigna unguiculata)

Dispositif expérimental : Le dispositif expérimental utilisé est celui des blocs complétement randomisés à trois répétitions. Une parcelle de $5 \mathrm{~m}^{2}$ a été divisée en 3 blocs distants les uns des autres de $0,5 \mathrm{~m}$. Chaque bloc représente chaque légumineuse et est constitué de 16 pots élémentaires chacun. Ces 16 pots sont disposés en trois groupes. Le premier groupe (G1) est constitué par une seule légumineuse (arachide, niébé ou soja). Le deuxième groupe (G2) est constitué par l'association de deux espèces (arachide-niébé, arachide-soja ou soja -niébé). Le troisième groupe (G3) est constitué des lots comportant l'association des trois variétés (arachideniébé-soja). Ces pots sont en réalité des sachets en polyéthylène noir de 80 microns d'épaisseur, de 25 $\mathrm{cm}$ de haut et $12 \mathrm{~cm}$ de large à plat. Ils sont remplis de terre à $7,5 \mathrm{~cm}$ de diamètre et $21 \mathrm{~cm}$ de haut, soit un volume d'un litre et un poids de $1,2 \mathrm{~kg}$ environ. Pour augmenter les chances de germination, trois graines de chaque espèce ont été semées par pot.
Toutes les graines ont été semées à $1 \mathrm{~cm}$ de profondeur. L'arrosage s'est fait chaque matin et chaque soir avec de l'eau de robinet. Le démariage a été fait dix jours après semis afin de conserver les meilleurs plants par pot. Un traitement d'insecticide avec cypercal 50 EC (cyperméthrine $50 \mathrm{~g} / 1)$ a été effectué 3 semaines après semis.

Collecte des données: Toutes les données ont été collectées à partir de la sixième semaine après semis correspondant au début des floraisons. Elles ont concernées le nombre de nodules, la longueur de la plante et celle du système racinaire, le poids de la plante et celui du système racinaire. Toutes les mesures ont été relevées sur trois pieds dans chaque groupe pris au hasard pour chaque espèce. La récolte a été faite avec précautions pour éviter la coupure des racines secondaires et la perte des nodules détachées des racines en débarrassant les plantes des sachets. Les racines des plantes ont été ensuite trempées dans de l'eau pour faciliter le 
comptage des nodules. Le nombre de nodules a été obtenu par simple comptage. La longueur de la plante a été déterminée par la mesure de la distance de la tige principale depuis le collet jusqu'à la feuille la plus extrême. Celle du système racinaire s'est faite du collet jusqu'au bout de la racine. Elles ont été mesurées à l'aide d'un ruban-mètre. Le système racinaire a été séparé de la plante et son poids frais ainsi que celui de la plante ont été déterminés à l'aide d'une balance Howell de précision $5 \mathrm{~kg} \times 1 \mathrm{~g}$.

\section{RESULTATS}

Effet de l'association des trois (3) espèces de légumineuses sur les paramètres agronomique de l'arachide: Les paramètres agronomiques tels que le nombre de nodules, la longueur et le poids de la plante, la longueur et le poids du système racinaire ont été estimés chez l'arachide cultivée seule ou en association. Les résultats obtenus ont permis de faire la distinction entre les associations car une différence significative a été observée entre elles pour ces variables (Tableau 1). Les résultats des analyses montrent que l'arachide cultivée seule enregistre les plus fortes valeurs du nombre de nodules $(72,66 \pm 12,89)$ et du poids de la plante $(94,80 \pm 7,74)$. De même, les nombres de nodules faibles ont été obtenus avec les associations de légumineuses (Figure 3). La valeur la plus faible au niveau du poids de la plante a été enregistrée au niveau de l'association arachide-soja-niébé $(31,80 \pm 11,47)$. Les associations arachide-niébé et arachide-soja ont donné des valeurs significativement identiques au niveau du poids de la plante. Les mesures faites sur la longueur du système racinaire ont montré qu'il n'existe pas de différence significative pour l'arachide cultivée seule et l'arachide en association. Par contre,
Analyse statistique des données: Les données recueillies pour chacune des 5 variables ont été saisies avec le tableur Excel version 97-2003 puis traitées à l'aide du logiciel STATISTICA édition version 7.1 à travers l'analyse de variance à deux facteurs (ANOVA). La signification du test a été déterminée en comparant la probabilité $(P)$ associée à la statistique au seuil $\alpha=0,05$. Lorsqu'une différence significative a été observée entre les caractères, l'ANOVA a été complétée par le test de la Plus Petite Différence Significative (PPDS).

le poids du système racinaire est plus élevé pour l'arachide sans association.

Effet de l'association des trois (3) espèces de légumineuses sur les paramètres agronomique du niébé : Le résultat de l'analyse statistique des données présenté par le Tableau 2 a montré que les variables telles que le nombre de nodules, la longueur et le poids de la plante, la longueur et le poids du système racinaire ont été influencées par l'association des légumineuses. La comparaison du nombre de nodule montre qu'il existe une différence significative entre le niébé planté seul et le niébé associé à l'arachide ainsi que dans l'association avec le soja et l'arachide $(P=0,00)$. Le nombre de nodule après culture est plus intense $(59,66 \pm 30,59)$, lorsque celui-ci n'est associé à aucune autre plante (Figure 4). Pour ce qui concerne la longueur et le poids de la plante, les plus fortes moyennes proviennent respectivement du niébé associé au soja $(19,76 \pm 2,35)$ et le niébé en culture pure $(46,13 \pm 18,94)$. L'étude du système racinaire, donne une différence significative $(P=0,00)$. De même le système racinaire s'intensifie quand le niébé n'est pas associé à une autre culture.

Tableau 1 : Analyse statistique des paramètres agronomiques mesurés sur l'arachide

\begin{tabular}{|c|c|c|c|c|c|c|}
\hline \multirow[b]{2}{*}{ Variables } & \multicolumn{4}{|c|}{ Moyennes (士écart-type) } & \multicolumn{2}{|c|}{ Statistiques } \\
\hline & A & AN & AS & ANS & $\mathbf{F}$ & $\mathbf{P}$ \\
\hline N. NOD & $72,66 \pm 12,89 \mathrm{c}$ & $59,00 \pm 40,03$ b & $57,66 \pm 28,58 \mathrm{~b}$ & $50,00 \pm 15,62 b$ & 26,86 & 0,00 \\
\hline L. $p(\mathrm{~cm})$ & $16,00 \pm 3,46$ a & $16,33 \pm 3,51^{\mathrm{a}}$ & $8,00 \pm 4,00^{a}$ & $14,33 \pm 5,50^{a}$ & 0,94 & 0,47 \\
\hline P. p (g) & $94,80 \pm 7,74$ e & $62,66 \pm 30,68 d$ & $51,06 \pm 38,53^{d}$ & $31,80 \pm 11,47^{b}$ & 16,42 & 0,00 \\
\hline L. SR (cm) & $29,66 \pm 2,51^{a}$ & $25,00 \pm 5,56^{a}$ & $34,00 \pm 9,53$ a & $29,33 \pm 12,85^{a}$ & 1,43 & 0,19 \\
\hline P. SR (g) & $16,26 \pm 1,61^{c}$ & $13,66 \pm 7,54 \mathrm{c}$ & $12,40 \pm 9,14 \mathrm{c}$ & $5,46 \pm 4,05$ b & 12,81 & 0,00 \\
\hline
\end{tabular}

Pour chaque variable, les valeurs portant les mêmes lettres sur la ligne sont statistiquement égales.

A : arachide, AN : arachide associée au niébé, AS : arachide associée au soja, ANS : arachide associée au niébé et au soja, N.NOD : Nombre de nodules, L.p. $(\mathrm{cm})$ : Longueur de la plante, P.p. (g) : Poids de la plante, L.SR $(\mathrm{cm})$ : Longueur du système racinaire, $P . S R(g)$ : Poids du système racinaire 


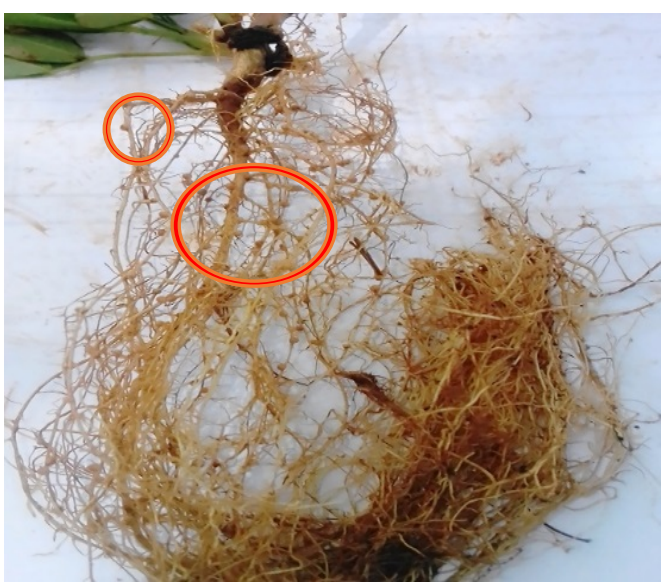

A : Arachide cultivée seule

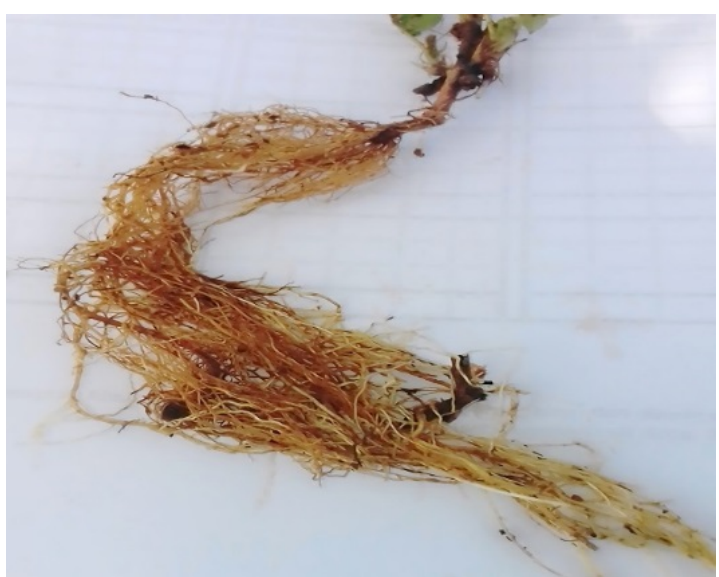

B : Arachide associée

Figure 3: Photographie montrant l'influence de l'association sur la capacité de nodulation de l'arachide

Tableau 2 : Analyse statistique des paramètres agronomiques mesurés sur le niébé

\begin{tabular}{lllllll} 
& \multicolumn{3}{c}{ Moyennes (técart-type) } & \multicolumn{2}{c}{ Statistiques } \\
\cline { 2 - 5 } Variables & N & NS & NA & NSA & F & P \\
\hline N. NOD & $59,66 \pm 30,59^{\mathrm{d}}$ & $50,66 \pm 22,94^{\mathrm{d}}$ & $9,00 \pm 2,00^{\mathrm{b}}$ & $24,33 \pm 11,23^{\mathrm{c}}$ & 2535,86 & 0,00 \\
L. p (cm) & $16,66 \pm 1,15^{\mathrm{c}}$ & $19,76 \pm 2,35^{\mathrm{e}}$ & $12,33 \pm 2,08^{\mathrm{c}}$ & $18,00 \pm 2,64^{\mathrm{d}}$ & 4,33 & 0,00 \\
P. p (g) & $46,13 \pm 18,94^{\mathrm{c}}$ & $8,70 \pm 3,08^{\mathrm{a}}$ & $23,20 \pm 15,81^{\mathrm{b}}$ & $41,00 \pm 24,51^{\mathrm{c}}$ & 2,66 & 0,01 \\
L. SR (cm) & $35,66 \pm 8,14^{\mathrm{e}}$ & $24,00 \pm 13,89^{\mathrm{c}}$ & $33,33 \pm 10,01^{\mathrm{d}}$ & $22,00 \pm 3,46^{\mathrm{c}}$ & 4,67 & 0,00 \\
P. SR (g) & $17,40 \pm 9,57^{\mathrm{e}}$ & $1,40 \pm 0,10^{\mathrm{a}}$ & $11,06 \pm 9,44^{\mathrm{d}}$ & $13,80 \pm 6,10^{\mathrm{d}}$ & 5,28 & 0,00 \\
\hline
\end{tabular}

Pour chaque variable, les valeurs portant les mêmes lettres sur la ligne sont statistiquement égales.

N : niébé, NA : niébé associé à l'arachide, NS : niébé associé au soja, NAS : niébé associé à l'arachide et au soja, N.NOD : Nombre de nodules, L.p. (cm) : Longueur de la plante, P.p. (g) : Poids de la plante, L.SR (cm) : Longueur du système racinaire, $\mathrm{P} . \mathrm{SR}(\mathbf{g})$ : Poids du système racinaire

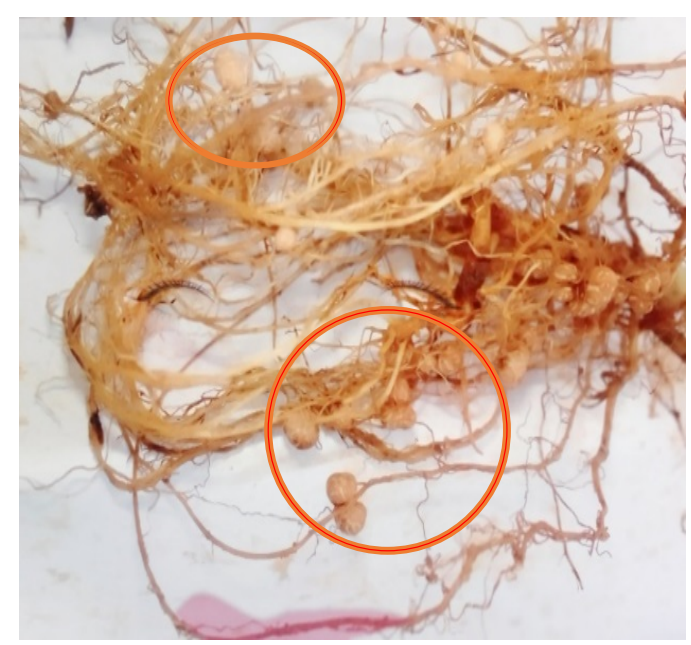

A : Niébé cultivé seul

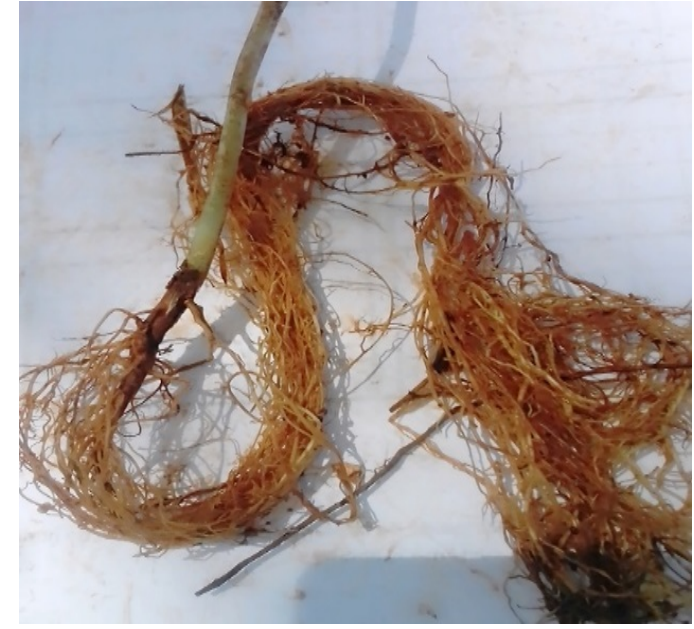

B : Niébé associé

Figure 4: Photographie montrant l'influence de l'association sur la capacité de nodulation du niébé

Effet de l'association des trois (3) espèces de légumineuses sur les paramètres agronomique du soja: L'analyse du Tableau 3 montre qu'il existe une différence significative entre les moyennes du soja cultivé seul et celles du soja associé à l'arachide et au niébé $(P=0,00)$. Cependant, les moyennes les plus élevées ne sont pas spécifiques à la culture pure du soja. Les nodules sont nombreux $(37,33 \pm 6,1)$, 
lorsque le soja est cultivé seul (Figure 5). De même, le soja grandit vite quand il est planté sans être associé $(29,66 \pm 3,5)$. Quant au poids de la plante, la plus forte moyenne a été obtenue lors de l'association du soja avec l'arachide $(34,60 \pm 10,6)$. Les racines du soja sont plus longues quand le soja est associé au niébé $(33,33 \pm 5,6)$. Par contre, c'est quand le soja est associé à l'arachide et au niébé que les racines donnent la meilleure moyenne $(5,86 \pm 6,3)$.

Tableau 3: Analyse statistique des paramètres agronomiques mesurés sur le soja

\begin{tabular}{lllllll} 
& \multicolumn{3}{c}{ Moyennes (técart-type) } & \multicolumn{2}{c}{ Statistiques } \\
\cline { 2 - 6 } Variables & $\mathbf{S}$ & SN & SA & SNA & F & P \\
\hline N. NOD & $37,33 \pm 6,1^{\mathrm{d}}$ & $22,66 \pm 11,5^{\mathrm{c}}$ & $6,33 \pm 2,3^{\mathrm{b}}$ & $22,00 \pm 25,9^{\mathrm{c}}$ & 11,9 & 0,0 \\
L. p (cm) & $29,66 \pm 3,5^{\mathrm{e}}$ & $24,00 \pm 1,7^{\mathrm{d}}$ & $20,00 \pm 3,6^{\mathrm{c}}$ & $19,33 \pm 1,1^{\mathrm{c}}$ & 5,1 & 0,0 \\
P. p (g) & $5,73 \pm 1,7^{\mathrm{b}}$ & $6,68 \pm 1,8^{\mathrm{c}}$ & $34,60 \pm 10,6^{\mathrm{f}}$ & $22,13 \pm 17,8^{\mathrm{d}}$ & 5,7 & 0,0 \\
L. SR (cm) & $32,33 \pm 13,6^{\mathrm{d}}$ & $33,33 \pm 5,6^{\mathrm{e}}$ & $21,00 \pm 3,6^{\mathrm{c}}$ & $26,00 \pm 10,3^{\mathrm{c}}$ & 4,5 & 0,0 \\
P. SR (g) & $1,53 \pm 0,6^{\mathrm{b}}$ & $1,11 \pm 0,2^{\mathrm{b}}$ & $4,26 \pm 0,7^{\mathrm{b}}$ & $5,86 \pm 6,3^{\mathrm{c}}$ & 4,7 & 0,0 \\
\hline P.
\end{tabular}

Pour chaque variable, les valeurs portant les mêmes lettres sur la ligne sont statistiquement égales.

S : soja, SA : soja associé à l'arachide, SN : soja associé au niébé, SNA : soja associé à l'arachide et au niébé, N.NOD : Nombre de nodules, L.p. (cm): Longueur de la plante, P.p. (g) : Poids de la plante, L.SR (cm) : Longueur du système racinaire, P.SR $(\mathrm{g})$ : Poids du système racinaire.

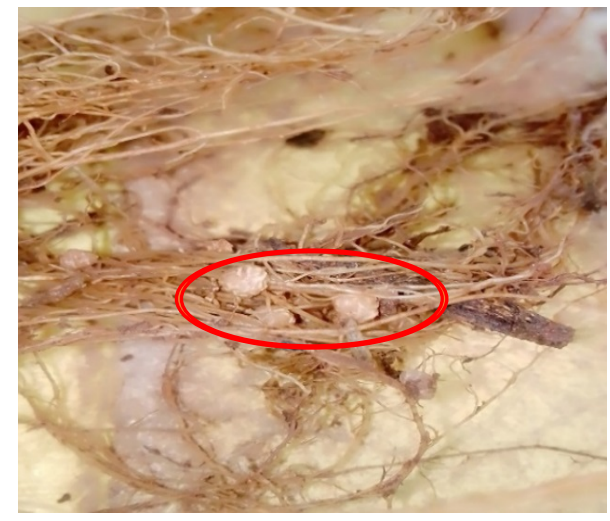

A : Soja cultivé seul

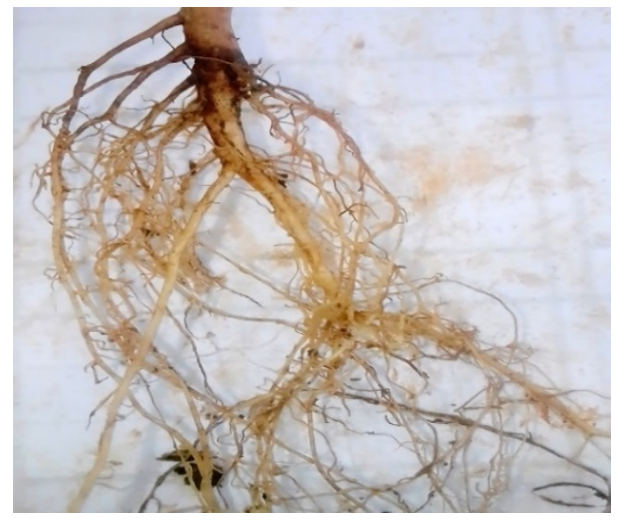

B : Soja associé

Figure 5: Photographie montrant l'influence de l'association sur la capacité de nodulation du soja

\section{DISCUSSION}

Le nombre de nodules a diminué lorsque les légumineuses sont cultivées en association. La plus grande valeur du nombre de nodules a été obtenue lorsque ces légumineuses sont en cultures pures. Cette augmentation significative du nombre de nodules pourrait être due au fait que la plante étant en culture pure utiliserait les ressources disponibles sans toutefois avoir de compétitions interspécifiques (Patrick, 1988). La baisse de la nodulation lorsque ces légumineuses sont associées serait due aux divers mécanismes en fonction des conditions environnementales. En effet, selon Tjepkema \& Winship (1980), la nodulation est non seulement un processus consommateur d'énergie mais nécessite également le contrôle strict d'un envahisseur bactérien. En conséquence, Gage (2004) a démontré que lorsque les conditions environnementales sont favorables, les racines qui sont susceptibles de nodulation permettront à certains filaments d'infection de se poursuivre alors que beaucoup d'autres seront arrêtés dans l'épiderme ou le cortex. De plus, après la formation des premiers primordia, l'initiation de nouveaux primordia sera inhibée, limitant ainsi le nombre de nodules. En outre, les conditions environnementales, telles que le manque de nitrate ou d'ammonium dans le sol, diminuent le processus de nodulation et de fixation de l'azote. La concurrence pour la lumière et les ressources disponibles dans le sol aurait influencé la plante en générale et plus spécifiquement sa capacité de nodulation. Dans cette même logique, Patrick (1988) affirme que l'association de plantes ne présentant pas de différences morphologiquement suffisant, n'offre pas d'intérêt et peut même se révéler néfaste à forte densité de peuplement. A cet effet, le feuillage, les ramifications des branches, ont une influence sur le 
bon fonctionnement de la plante et sur sa nodulation. Aussi, les plantes mises en association ne présentent pas les mêmes cycles végétatifs, donc les dates de floraison différentes, date à laquelle les résultats ont été recueillis. Ainsi, nos résultats ont montré que parmi les trois légumineuses étudiées (arachide, niébé et soja), la plus forte nodulation a été observée chez l'arachide. Cela s'expliquerait par la différence entre les cycles de cultures de ces trois légumineuses. Dans notre étude, la longueur et le poids de la plante les plus importants ont été obtenus lorsque les légumineuses sont en cultures pures.

\section{CONCLUSION ET PERSPECTIVES}

L'objectif de notre étude visait à promouvoir la pratique culturale pouvant améliorer la capacité de nodulation des légumineuses. Dans notre étude, les résultats ont montré que l'association des légumineuses a un impact néfaste sur la capacité de nodulation de celles-ci. II ressort donc de cette étude

\section{REMERCIEMENTS}

Nos remerciements vont à l'endroit des autorités de l'université Jean Lorougnon GUEDE et des Enseignants du laboratoire de l'amélioration et de la

\section{REFERENCES}

Akakpo C., Amadji F. \& Carsky R.J. (1999). Intégration du mucuna dans les systèmes culturaux du sud Bénin, In : R. J.Carsky, A. C Etéka, J. D. H. Keatinge \& V. M. Manyong (éditeurs), Plantes de couverture et gestion des ressources naturelles en Afrique occidentale, Actes de l'atelier, Octobre, 1999, lita, Ciepca, Cotonou (Benin) : 175184.

Atlas (2007). Le département de Daloa. Géologie et pédologie, $12 \mathrm{p}$.

Ba I. (2014). Recensement Général de la Population et de l'Habitat, Rapport d'exécution et Présentation des principaux résultats, Cote d'Ivoire, $49 \mathrm{p}$.

Baudoin JP., Camarena F., Lobo M. \& Mergeai G. (2001). Breeding phaseolus for intercrop combinations in Andean highlands. In HD. Cooper, C. Spillane,T. Hodgkin (Eds). Broadening the genetic bases of crop. Wallingford, UK: CAB International, 373-384 pp.

Carsky R.J., Douthwaite B., Manyong V.M., Sanginga N., Schulz S., Vanlauwe B., Diels J. \& Keatinge J.D.H. (2003). Amélioration de la gestion des sols par l'introduction de légumineuses dans les systèmes céréaliers
Cela pourrait s'expliquer par l'effet de compétition des ressources disponibles dans le sol empêchant ainsi la croissance des plantes. Quant à la longueur et au poids du système racinaire, les valeurs les plus importantes ont été obtenues lorsque deux légumineuses sont associées. Ces résultats corroborent ceux de Louarn et al., (2010). Pour ces auteurs, ces résultats seraient dus au phénomène de facilitation, phénomène au cours duquel une espèce améliore les conditions environnementales de développement de l'espèce associée (température, ombre et disponibilité des ressources).

que les légumineuses cultivées en cultures pures ont permis l'optimisation du rendement des nodules. Aussi, cette présente étude pourrait être étendue à d'autres légumineuses en prenant en compte la fertilisation organique, l'écartement entre les pieds de légumineuses et la densité de semi de celles-ci.

production végétale pour les efforts consentis dans la réalisation de ces travaux.

des savanes africaines. Cahiers Agricultures, $12: 227-233$

Gage D.J. (2004). Infection and invasion of roots by symbiotic, nitrogen-fixing rhizobia during nodulation of temperate legumes. Microbiology and Molecular Biology Reviews 68, 280-300.

Louarn, G., Corre-Hellou G., Fustec J., Lô-Pelzer E., Julier B., Litrico I., Hinsinger P., Lecomte C. (2010). Déterminants écologiques et physiologiques de la productivité et de la stabilité des associations graminéeslégumineuses. Innovations Agronomiques, 11, pp.79-99. Manyong (éditeurs), Plantes de couverture et gestion des ressources naturelles en Afrique occidentale Actes de l'atelier, Octobre, 1999, lita, Ciepca, Cotonou (Benin) : 175-184.

Patrick S. (1998). Amélioration de systèmes de cultures associées céréale-légumineuse au Cameroun, Thèse de Doctorat-Ingénieur en Agronomie, Ecole Normale Supérieure Agronomique de Montpellier, France, $229 \mathrm{p}$.

TAffouo V.D., Ndongo D. J.E., Nguelemeni M.P., Eyambe Y.M., Tayou R.F. \& Akoa A. (2008). Effets de la densité de semis sur la croissance, le rendement et les teneurs en composés organiques chez cinq variétés de 
Kouame et al., J. Appl. Biosci. 2020 Influence de l'association culturale sur la capacité de nodulation de trois espèces de légumineuses : Arachide, Niébé et Soja vert

niébé Vigna unguiculata (L).Walp). Journal of Applied Biosciences, 12: 623-632.

Tjepkema J.D. \& Winship L.J. (1980). Energy requirement for nitrogen fixation in actinorhizal and legume root nodules. Science 209, 279-281. 